\title{
A TABLE OF ELLIPTIC INTEGRALS: TWO QUADRATIC FACTORS
}

\author{
B. C. CARLSON
}

\begin{abstract}
Thirteen integrands that are rational except for the square root of a quartic polynomial with two pairs of conjugate complex zeros are integrated in terms of $R$-functions of real variables. In contrast with previous tables, the formulas hold for all real intervals of integration for which the integrals exist (possibly as Cauchy principal values). This is achieved by using Landen's transformation and the duplication theorem. In an appendix, an elliptic integral of the third kind with a restricted complex parameter is transformed to make the parameter real. Also, a degenerate integral of the first kind is separated into real and imaginary parts.
\end{abstract}

\section{INTRODUCTION}

This paper treats integrands that are rational except for the square root of a quartic polynomial with two pairs of conjugate complex zeros. Integrals of the form

$$
[p]=\left[p_{1}, \ldots, p_{5}\right]=\int_{y}^{x} \prod_{i=1}^{5}\left(a_{i}+b_{i} t\right)^{p_{i} / 2} d t,
$$

where $p_{1}, \ldots, p_{4}$ are odd integers and $p_{5}$ is even, are treated in $[4,5]$ if all quantities are real. Reference [8] deals with cases where $p_{2}=p_{3}$ and $a_{3}+b_{3} t$ is the complex conjugate of $a_{2}+b_{2} t$. Here we assume further that $p_{1}=p_{4}$ and $a_{4}+b_{4} t$ is the complex conjugate of $a_{1}+b_{1} t$. That is, we consider

$$
\left[p_{1}, p_{2}, p_{2}, p_{1}, p_{5}\right]=\int_{y}^{x} \prod_{i=1}^{2}\left(f_{i}+g_{i} t+h_{i} t^{2}\right)^{p_{i} / 2}\left(a_{5}+b_{5} t\right)^{p_{5} / 2} d t,
$$

where all quantities are real, $x>y, f_{i}+g_{i} t+h_{i} t^{2}>0$ for all real $t, p_{1}$ and $p_{2}$ are odd integers, and $p_{5}$ is even. We retain the redundant notation on the left side of (1.2), omitting $p_{5}$ if it is 0 , for consistency with [5,8]. Section 2 contains the 11 cases (apart from exchange of $p_{1}$ and $p_{2}$ ) with $2\left|p_{1}\right|+2\left|p_{2}\right|+\left|p_{5}\right| \leq 8$ and $2 p_{1}+2 p_{2}+p_{5} \leq 0$, as well as $[1,1,1,1,-2]$ and $[1,1,1,1]$. The formulas hold for all $x$ and $y$ for which the integral exists (possibly as a Cauchy principal value if $p_{5}=-2$ ).

Received by the editor June 26, 1991.

1991 Mathematics Subject Classification. Primary 33E05; Secondary 33C75.

This work was supported by the Director of Energy Research, Office of Basic Energy Sciences. The Ames Laboratory is operated for the U.S. Department of Energy by Iowa State University under contract W-7405-ENG-82. 
In Byrd and Friedman's table [1, $\S 267]$ such integrals are listed only with a lower limit that depends on the parameters in the integrand, and a restriction on the upper limit is added in $[9,3.145(4)]$ so that $\phi$ in Legendre's $F(\phi, k)$ is between 0 and $\pi / 2$. Also, there is an ambiguity of sign; e.g., [1, 267.00] with $a_{1}=a_{2}=\sqrt{2}$ is correct if $b_{2}=-b_{1}=1 / 2$ and $y=1$ but incorrect if $b_{1}=-b_{2}=1 / 2$ and $y=2$ unless $g_{1}$ is taken to be the negative square root of $g_{1}^{2}$.

The integrals (1.2) are expressed in terms of four $R$-functions:

$$
\begin{gathered}
R_{F}(x, y, z)=\frac{1}{2} \int_{0}^{\infty}[(t+x)(t+y)(t+z)]^{-1 / 2} d t \\
R_{J}(x, y, z, w)=\frac{3}{2} \int_{0}^{\infty}[(t+x)(t+y)(t+z)]^{-1 / 2}(t+w)^{-1} d t
\end{gathered}
$$

and two special cases,

$$
R_{D}(x, y, z)=R_{J}(x, y, z, z)
$$

and

$$
R_{C}(x, y)=R_{F}(x, y, y)=\frac{1}{2} \int_{0}^{\infty}(t+x)^{-1 / 2}(t+y)^{-1} d t .
$$

The functions $R_{F}, R_{D}$, and $R_{J}$ respectively replace Legendre's elliptic integrals of the first, second, and third kinds, while $R_{C}$, which requires special attention in this paper, includes the inverse circular (if $0 \leq x<y$ ) and inverse hyperbolic (if $0<y<x$ ) functions. Fortran codes for numerical computation of all four functions are listed in the Supplements to $[4,5]$ and are available in several major software libraries.

In [8] a Landen transformation was used to change the first two variables of $R_{F}, R_{D}$, and $R_{J}$ from complex to real numbers; the remaining variables, including those of $R_{C}$, were never complex. In the present paper the complex variables are the parameter (the fourth variable) of $R_{J}$ and both variables of $R_{C}$. However, a Landen transformation of $R_{F}$ and $R_{D}$ is used in $\S 3$ to eliminate a restriction on the interval of integration that arose in [3] because of a branch point. In $\S 4$ the complex parameter of $R_{J}$ is made real, not by a direct Landen transformation but by an inverse Landen transformation followed by the duplication theorem (see Appendix A), a combination that also takes care of the branch-point problem. The function $R_{C}$ with complex variables is separated into real and imaginary parts in Appendix $\mathrm{B}$, and the imaginary part cancels another $R_{C}$ that comes from the inverse Landen transformation of $R_{J}$.

The formulas of $[5,8]$ made it unnecessary to do any further work with recurrence relations, although conversion to notation appropriate for this paper sometimes entailed tedious algebra. The integrals $I_{2}, I_{3}$, and $I_{3}^{\prime}$ used previously are now complex, but the eventual cancellation of imaginary terms provided a partial check. The 13 integral formulas in $\S 2$ were checked by numerical integration; some details of the checks are given in $\S 5$. The variables of $R_{J}$ and $R_{C}$ are nonnegative, even when the three integrals with $p_{5}=-2$ have their Cauchy principal values.

\section{TABLE OF INTEGRALS}

We assume $x>y$ and $f_{i}+g_{i} t+h_{i} t^{2}>0$ for $i=1,2$ and all real $t$. Some relations useful for numerical checks are included among the following 
definitions:

$$
\begin{gathered}
\xi_{i}=\left(f_{i}+g_{i} x+h_{i} x^{2}\right)^{1 / 2}, \quad \eta_{i}=\left(f_{i}+g_{i} y+h_{i} y^{2}\right)^{1 / 2}, \\
\xi_{1}^{\prime}=\left(g_{1}+2 h_{1} x\right) / 2 \xi_{1}, \quad \eta_{1}^{\prime}=\left(g_{1}+2 h_{1} y\right) / 2 \eta_{1}, \\
B=\xi_{1}^{\prime} \xi_{2}-\eta_{1}^{\prime} \eta_{2}, \quad E=\xi_{1}^{\prime} \xi_{1}^{2} \xi_{2}-\eta_{1}^{\prime} \eta_{1}^{2} \eta_{2}, \\
\theta_{i}=2 f_{i}+g_{i}(x+y)+2 h_{i} x y=\xi_{i}^{2}+\eta_{i}^{2}-h_{i}(x-y)^{2}, \\
\zeta_{i}=\left(2 \xi_{i} \eta_{i}+\theta_{i}\right)^{1 / 2}=\left[\left(\xi_{i}+\eta_{i}\right)^{2}-h_{i}(x-y)^{2}\right]^{1 / 2}, \\
U=\left(\xi_{1} \eta_{2}+\eta_{1} \xi_{2}\right) /(x-y), \quad M=\zeta_{1} \zeta_{2} /(x-y), \\
\delta_{i j}=\left(2 f_{i} h_{j}+2 f_{j} h_{i}-g_{i} g_{j}\right)^{1 / 2}, \quad \Delta=\left(\delta_{12}^{4}-\delta_{11}^{2} \delta_{22}^{2}\right)^{1 / 2}, \\
\Delta_{ \pm}=\delta_{12}^{2} \pm \Delta, \quad L_{ \pm}^{2}=M^{2}+\Delta_{ \pm}, \quad L_{+} L_{-}=2 M U \\
G=2 \Delta \Delta_{+} R_{D}\left(M^{2}, L_{-}^{2}, L_{+}^{2}\right) / 3+\Delta / 2 U \\
+\left(\delta_{12}^{2} \theta_{1}-\delta_{11}^{2} \theta_{2}\right) / 4 \xi_{1} \eta_{1} U
\end{gathered}
$$

For integrals with $p_{5} \neq 0$ we also define

$$
\begin{aligned}
& \alpha_{i 5}=2 f_{i} b_{5}-g_{i} a_{5}, \quad \beta_{i 5}=g_{i} b_{5}-2 h_{i} a_{5}, \\
& \gamma_{i}=\left(\alpha_{i 5} b_{5}-\beta_{i 5} a_{5}\right) / 2=f_{i} b_{5}^{2}-g_{i} a_{5} b_{5}+h_{i} a_{5}^{2}>0 \text {, } \\
& \Lambda=\delta_{11}^{2} \gamma_{2} / \gamma_{1}, \quad \Omega^{2}=M^{2}+\Lambda, \\
& \psi=\left(\alpha_{15} \beta_{25}-\alpha_{25} \beta_{15}\right) / 2 \\
& =\left(g_{1} h_{2}-g_{2} h_{1}\right) a_{5}^{2}-2\left(f_{1} h_{2}-f_{2} h_{1}\right) a_{5} b_{5}+\left(f_{1} g_{2}-f_{2} g_{1}\right) b_{5}^{2}, \\
& \psi^{2}=-\delta_{11}^{2} \gamma_{2}^{2}+2 \delta_{12}^{2} \gamma_{1} \gamma_{2}-\delta_{22}^{2} \gamma_{1}^{2}=\gamma_{1} \gamma_{2}\left(\Delta_{+}-\Lambda\right)\left(\Lambda-\Delta_{-}\right) / \Lambda \text {, } \\
& \xi_{5}=a_{5}+b_{5} x, \quad \eta_{5}=a_{5}+b_{5} y, \\
& A\left(p_{1}, p_{2}, p_{2}, p_{1}, p_{5}\right)=\xi_{1}^{p_{1}} \xi_{2}^{p_{2}} \xi_{5}^{p_{5} / 2}-\eta_{1}^{p_{1}} \eta_{2}^{p_{2}} \eta_{5}^{p_{5} / 2} \text {, } \\
& X=\left[\xi_{5}\left(\alpha_{15}+\beta_{15} y\right) \eta_{2} / \eta_{1}+\eta_{5}\left(\alpha_{15}+\beta_{15} x\right) \xi_{2} / \xi_{1}\right] / 2(x-y) \\
& =\xi_{5} \eta_{5}\left[\theta_{1} A(-1,1,1,-1) / 2-\xi_{5} \eta_{5} A(1,1,1,1,-4)\right] /(x-y)^{2} \text {, } \\
& S=\left(M^{2}+\delta_{12}^{2}\right) / 2-U^{2}=\left(\xi_{1} \eta_{1} \theta_{2}+\xi_{2} \eta_{2} \theta_{1}\right) /(x-y)^{2}, \\
& \mu=\gamma_{1} \xi_{5} \eta_{5} / \xi_{1} \eta_{1}, \quad T=\mu S+2 \gamma_{1} \gamma_{2}, \quad V^{2}=\mu^{2}\left(S^{2}+\Lambda U^{2}\right), \\
& a=S \Omega^{2} / U+2 \Lambda U, \quad b^{2}=\left(S^{2} / U^{2}+\Lambda\right) \Omega^{4}, \\
& a^{2}=b^{2}+\Lambda^{2} \psi^{2} / \gamma_{1} \gamma_{2}=b^{2}+\Lambda\left(\Delta_{+}-\Lambda\right)\left(\Lambda-\Delta_{-}\right) \text {, } \\
& H=\delta_{11}^{2} \psi\left[R_{J}\left(M^{2}, L_{-}^{2}, L_{+}^{2}, \Omega^{2}\right) / 3+R_{C}\left(a^{2}, b^{2}\right) / 2\right] / \gamma_{1}^{2} \\
& -X R_{C}\left(T^{2}, V^{2}\right) \text {. }
\end{aligned}
$$

We shall want some of the quantities above when $a_{5}=1$ and $b_{5}=0$. These will be labeled by a subscript 0 :

$$
\Lambda_{0}=\delta_{11}^{2} h_{2} / h_{1}, \quad \Omega_{0}^{2}=M^{2}+\Lambda_{0},
$$




$$
\begin{aligned}
& \psi_{0}=g_{1} h_{2}-g_{2} h_{1} \\
& \psi_{0}^{2}=-\delta_{11}^{2} h_{2}^{2}+2 \delta_{12}^{2} h_{1} h_{2}-\delta_{22}^{2} h_{1}^{2}=h_{1} h_{2}\left(\Delta_{+}-\Lambda_{0}\right)\left(\Lambda_{0}-\Delta_{-}\right) / \Lambda_{0}, \\
& X_{0}=-\left(\xi_{1}^{\prime} \xi_{2}+\eta_{1}^{\prime} \eta_{2}\right) /(x-y) \\
& =\left[\theta_{1} A(-1,1,1,-1) / 2-A(1,1,1,1)\right] /(x-y)^{2}, \\
& \text { (2.26) } \quad \mu_{0}=h_{1} / \xi_{1} \eta_{1}, \quad T_{0}=\mu_{0} S+2 h_{1} h_{2}, \quad V_{0}^{2}=\mu_{0}^{2}\left(S^{2}+\Lambda_{0} U^{2}\right) \text {, } \\
& \text { (2.27) } a_{0}=S \Omega_{0}^{2} / U+2 \Lambda_{0} U, \quad b_{0}^{2}=\left(S^{2} / U^{2}+\Lambda_{0}\right) \Omega_{0}^{4} \text {, } \\
& \text { (2.28) } \quad a_{0}^{2}=b_{0}^{2}+\Lambda_{0}^{2} \psi_{0}^{2} / h_{1} h_{2}=b_{0}^{2}+\Lambda_{0}\left(\Delta_{+}-\Lambda_{0}\right)\left(\Lambda_{0}-\Delta_{-}\right) \text {, } \\
& H_{0}=\delta_{11}^{2} \psi_{0}\left[R_{J}\left(M^{2}, L_{-}^{2}, L_{+}^{2}, \Omega_{0}^{2}\right) / 3+R_{C}\left(a_{0}^{2}, b_{0}^{2}\right) / 2\right] / h_{1}^{2} \\
& -X_{0} R_{C}\left(T_{0}^{2}, V_{0}^{2}\right) \text {. }
\end{aligned}
$$

If the interval of integration is infinite, convergent integrals (with $2 p_{1}+2 p_{2}+$ $\left.p_{5} \leq-4\right)$ do not involve $H_{0}$. If $x \rightarrow+\infty$ and $y$ is finite, we find (for $i=1,2$ ) that

$$
\begin{array}{rlrl}
\xi_{i} & \sim h_{i}^{1 / 2} x, & \theta_{i} & \sim\left(g_{i}+2 h_{i} y\right) x, \\
U=h_{1}^{1 / 2} \eta_{2}+h_{2}^{1 / 2} \eta_{1}, & M^{2}=\prod_{i=1}^{2}\left(2 h_{i}^{1 / 2} \eta_{i}+g_{i}+2 h_{i} y\right) .
\end{array}
$$

If $y \rightarrow-\infty$ and $x$ is finite, then (for $i=1,2$ )

$$
\begin{array}{rlrl}
\eta_{i} & \sim h_{i}^{1 / 2}|y|, & \theta_{i} & \sim\left(g_{i}+2 h_{i} x\right) y, \\
U=h_{1}^{1 / 2} \xi_{2}+h_{2}^{1 / 2} \xi_{1}, & M^{2}=\prod_{i=1}^{2}\left(2 h_{i}^{1 / 2} \xi_{i}-g_{i}-2 h_{i} x\right) .
\end{array}
$$

If $x=-y \rightarrow+\infty$, then (for $i=1,2$ )

$$
\begin{gathered}
\xi_{i} \sim \eta_{i} \sim h_{i}^{1 / 2} x, \quad \theta_{i} \sim-2 h_{i} x^{2}, \quad \zeta_{i}=\delta_{i i} / h_{i}^{1 / 2}, \\
1 / U=M=R_{C}\left(a^{2}, b^{2}\right)=X R_{C}\left(T^{2}, V^{2}\right)=0 .
\end{gathered}
$$

In all three of the limiting cases an identity useful for (2.41) is

$$
\begin{aligned}
& B-b_{5} A(1,1,1,1,-2) \\
& \quad=\left(\alpha_{15}+\beta_{15} y\right) \eta_{2} / 2 \eta_{1} \eta_{5}-\left(\alpha_{15}+\beta_{15} x\right) \xi_{2} / 2 \xi_{1} \xi_{5} .
\end{aligned}
$$

Aside from interchange of $p_{1}$ and $p_{2}$, there are 11 integrals

$$
\left[p_{1}, p_{2}, p_{2}, p_{1}, p_{5}\right]=\int_{y}^{x} \prod_{i=1}^{2}\left(f_{i}+g_{i} t+h_{i} t^{2}\right)^{p_{i} / 2}\left(a_{5}+b_{5} t\right)^{p_{5} / 2} d t
$$

with odd integers $p_{1}, p_{2}$ and even integer $p_{5}$ such that $2\left|p_{1}\right|+2\left|p_{2}\right|+\left|p_{5}\right| \leq 8$ and $2 p_{1}+2 p_{2}+p_{5} \leq 0$. We shall include also $[1,1,1,1,-2]$ and $[1,1,1,1]$. The integral of the first kind is

$$
[-1,-1,-1,-1]=4 R_{F},
$$

and the next two integrals are of the second kind:

$$
[-3,1,1,-3]=4\left(-G+\Delta_{+} R_{F}\right) / \delta_{11}^{2},
$$




$$
\begin{aligned}
{[-3,-1,-1,-3]=} & 8 h_{1}\left[\left(\Lambda_{0}-\delta_{12}^{2}\right) G / \Delta-\left(\Lambda_{0}-\Delta_{+}\right) R_{F}\right] / \delta_{11}^{2} \Delta \\
& -4 \psi_{0} A(-1,1,1,-1) / \Delta^{2} .
\end{aligned}
$$

Like the three preceding integrals, three integrals of the third kind with $2 p_{1}+$ $2 p_{2}+p_{5} \leq-4$ are not restricted to finite intervals of integration. They involve $H$ but not $H_{0}$ :

$$
\begin{aligned}
& {[-1,-1,-1,-1,-2]=-2\left(b_{5} H+\beta_{15} R_{F} / \gamma_{1}\right),} \\
& {[1,-1,-1,1,-4]} \\
& =\left[\psi H+G+\left(\Lambda-\Delta_{+}\right) R_{F}\right] / \gamma_{2} \\
& \quad-\left[\beta_{15} A(-1,1,1,-1)+2 \gamma_{1} A(-1,1,1,-1,-2)\right] / 2 b_{5} \gamma_{2}, \\
& {[-1,-1,-1,-1,-4]=b_{5}\left(\beta_{15} / \gamma_{1}+\beta_{25} / \gamma_{2}\right) H+\beta_{15}^{2} R_{F} / \gamma_{1}^{2}} \\
& \quad+b_{5}^{2}\left[\Sigma-b_{5} A(1,1,1,1,-2)\right] / \gamma_{1} \gamma_{2} .
\end{aligned}
$$

Seven integrals of the third kind have $2 p_{1}+2 p_{2}+p_{5} \geq-2$ and exist only for finite intervals of integration. Four of them with $p_{5} \geq 0$ involve $H_{0}$ but not $H$ :

$$
\begin{gathered}
{[-1,-1,-1,-1,2]=2 b_{5} H_{0}-2 \beta_{15} R_{F} / h_{1}} \\
{[1,-1,-1,1]=\left(\psi_{0} H_{0}+\Sigma+\Lambda_{0} R_{F}\right) / h_{2}} \\
{[-1,-1,-1,-1,4]=-b_{5}\left(\beta_{15} / h_{1}+\beta_{25} / h_{2}\right) H_{0}} \\
+b_{5}^{2} \Sigma / h_{1} h_{2}+\beta_{15}^{2} R_{F} / h_{1}^{2} \\
{[1,1,1,1]=\left(\delta_{22}^{2} / h_{2}^{2}-\delta_{11}^{2} / h_{1}^{2}\right)\left[\psi_{0} H_{0}+\left(\Lambda_{0}-\delta_{12}^{2}\right) R_{F}\right] / 8} \\
-\left(3 \psi_{0}^{2}-4 h_{1} h_{2} \delta_{12}^{2}\right)\left(\Sigma+\delta_{12}^{2} R_{F}\right) / 24 h_{1}^{2} h_{2}^{2} \\
+\left[\Delta^{2} R_{F}-\psi_{0} A(1,1,1,1)\right] / 12 h_{1} h_{2}+E / 3 h_{1} .
\end{gathered}
$$

The final three integrals have $p_{5}<0$ and involve both $H$ and $H_{0}$ :

$$
[1,-1,-1,1,-2]=2\left(-\gamma_{1} H+h_{1} H_{0}\right) / b_{5},
$$

$$
\begin{aligned}
{[1,1,1,1,-2]=} & -2 \gamma_{1} \gamma_{2} H / b_{5}^{3}+\left[\left(h_{1} \gamma_{2}+h_{2} \gamma_{1}\right) / b_{5}^{3}-\psi_{0}^{2} / 4 h_{1} h_{2} b_{5}\right] H_{0} \\
& +\left(\beta_{15} / h_{1}+\beta_{25} / h_{2}\right)\left(\Sigma+\Lambda_{0} R_{F}\right) / 4 b_{5}^{2} \\
& -\delta_{11}^{2} \psi_{0} R_{F} / 2 h_{1}^{2} b_{5}+A(1,1,1,1) / 2 b_{5} \\
{[1,1,1,1,-4]=} & \left.-\left(\gamma_{1} \beta_{25}+\gamma_{2} \beta_{15}\right) H+\left(h_{1} \beta_{25}+h_{2} \beta_{15}\right) H_{0}\right] / b_{5}^{3} \\
& \left.+\left[2 \Sigma+\left(\Lambda+\Lambda_{0}\right) R_{F}\right)\right] / b_{5}^{2}-A(1,1,1,1,-2) / b_{5}
\end{aligned}
$$

\section{INTEGRALS OF THE FIRST AND SECOND KINDS}

In this section we derive (2.36), (2.37), and (2.38). By [5, (2.13), (2.17)],

$$
\begin{gathered}
I_{1}=\int_{y}^{x} \prod_{i=1}^{4}\left(a_{i}+b_{i} t\right)^{-1 / 2} d t=2 R_{F}\left(U_{12}^{2}, U_{13}^{2}, U_{14}^{2}\right), \\
(x-y) U_{i j}=X_{i} X_{j} Y_{k} Y_{m}+Y_{i} Y_{j} X_{k} X_{m}, \\
X_{i}=\left(a_{i}+b_{i} x\right)^{1 / 2}, \quad Y_{i}=\left(a_{i}+b_{i} y\right)^{1 / 2}
\end{gathered}
$$


where $i, j, k, m$ is any permutation of $1,2,3,4$. Let

$$
\begin{aligned}
& \left(a_{1}+b_{1} t\right)\left(a_{4}+b_{4} t\right)=f_{1}+g_{1} t+h_{1} t^{2}>0, \quad-\infty<t<\infty, \\
& \left(a_{2}+b_{2} t\right)\left(a_{3}+b_{3} t\right)=f_{2}+g_{2} t+h_{2} t^{2}>0, \quad-\infty<t<\infty .
\end{aligned}
$$

Then the biquadratic polynomial in the integrand of

$$
I_{1}=\int_{y}^{x}\left[\left(f_{1}+g_{1} t+h_{1} t^{2}\right)\left(f_{2}+g_{2} t+h_{2} t^{2}\right)\right]^{-1 / 2} d t
$$

has two pairs of conjugate complex zeros. Equation (3.1) remains valid by the permanence of functional relations if the $U_{i j}$ are in the open right half-plane. We shall see that $U_{14}>0$, and we may choose $U_{13}>0$. Although $U_{12}$ is real, it may be negative, and (3.1) is then invalid if $R_{F}$ is taken to have the principal value represented by the integral (1.3). The reason is that $R_{F}$, as a function of any one of its variables, has a branch point at the origin $[2, \S 8.3]$. When $U_{12}$ describes a semicircle about 0 from the positive to the negative real axis, $U_{12}^{2}$ makes a complete circle and $R_{F}$ returns to a different branch. Negative values of $U_{12}$ may occur, as shown in [3, $\S 4$, when the quadrilateral whose vertices are the complex zeros has diagonals intersecting at an interior point of the interval of integration. The integral $I_{1}$ can then be expressed in terms of two standard integrals by breaking the interval of integration at the intersection. In the present paper we shall eliminate this complication by using Landen's transformation [7, (5.5)] to write

$$
\begin{gathered}
I_{1}=4 R_{F}\left(M^{2}, L_{-}^{2}, L_{+}^{2}\right), \quad M=U_{12}+U_{13}, \\
L_{ \pm}=\left[\left(U_{14}+U_{12}\right)\left(U_{14}+U_{13}\right)\right]^{1 / 2} \pm\left[\left(U_{14}-U_{12}\right)\left(U_{14}-U_{13}\right)\right]^{1 / 2}, \\
L_{+} L_{-}=2 M U_{14}, \quad L_{ \pm}^{2}-M^{2}=\left[\left(U_{14}^{2}-U_{12}^{2}\right)^{1 / 2} \pm\left(U_{14}^{2}-U_{13}^{2}\right)^{1 / 2}\right]^{2},
\end{gathered}
$$

where $M, L_{-}$, and $L_{+}$will be proved nonnegative for every interval of integration. Alternatively, the duplication theorem could be used for the same purpose, but the resulting expressions are less simple.

In (3.2), since only $f_{i}, g_{i}$, and $h_{i}$ are given, we may choose $b_{1}=b_{4}=h_{1}^{1 / 2}>$ $0, b_{2}=b_{3}=h_{2}^{1 / 2}>0, \operatorname{Im}\left(a_{1}\right)>0$, and $\operatorname{Im}\left(a_{2}\right)>0$. If we assume $x$ and $y$ to be finite and take the principal branch of the square roots in (3.1), then $X_{1}, Y_{1}, X_{2}, Y_{2}$ lie in the open first quadrant of the complex plane, while their respective complex conjugates $X_{4}, Y_{4}, X_{3}, Y_{3}$ lie in the open fourth quadrant. It is clear that $U_{14}>0$ because both terms of $(x-y) U_{14}$ are strictly positive and we assume $x>y$. The same assumption, along with $\operatorname{Im}\left(a_{1}\right)>0$, shows that

$$
\operatorname{Im}\left(X_{1}^{2} Y_{4}^{2}\right)=\operatorname{Im}\left[\left(a_{1}+b_{1} x\right)\left(a_{4}+b_{4} y\right)\right]=h_{1}^{1 / 2}(y-x) \operatorname{Im}\left(a_{1}\right)<0 .
$$

Thus, $X_{1} Y_{4}$ is in the open fourth quadrant if $x$ and $y$ are finite, and a similar argument shows that $X_{3} Y_{2}$ is in the open first quadrant. Hence the product $X_{1} X_{3} Y_{2} Y_{4}$ is in the open right half-plane, its real part is positive, and $U_{13}>0$. Because $X_{2} Y_{3}$ is the complex conjugate of $X_{3} Y_{2}$ and so is in the open fourth quadrant, $X_{1} X_{2} Y_{3} Y_{4}$ is in the open lower half-plane, and $U_{12}$ may be positive or negative. However, $X_{1} Y_{4}+Y_{1} X_{4}$ and $X_{2} Y_{3}+Y_{2} X_{3}$ are both strictly positive, whence

$$
U_{12}+U_{13}=\left(X_{1} Y_{4}+Y_{1} X_{4}\right)\left(X_{2} Y_{3}+Y_{2} X_{3}\right) /(x-y)>0
$$


It follows from (3.1) that

$$
U_{i k}^{2}-U_{i m}^{2}=d_{i j} d_{k m}, \quad d_{i j}=a_{i} b_{j}-a_{j} b_{i} .
$$

In particular, we see that

$$
\begin{aligned}
& U_{14}^{2}-U_{13}^{2}=d_{12} d_{43}=\left|d_{12}\right|^{2}>0, \\
& U_{13}^{2}-U_{12}^{2}=d_{14} d_{32}>0, \\
& U_{14}^{2}-U_{12}^{2}=d_{13} d_{42}=\left|d_{13}\right|^{2}>0 .
\end{aligned}
$$

Note that $d_{12} \neq 0$ because we exclude the degenerate case where $a_{1}+b_{1} t$ is proportional to $a_{2}+b_{2} t$ (whence $f_{1}+g_{1} t+h_{1} t^{2}$ is proportional to $f_{2}+g_{2} t+h_{2} t^{2}$, and the integral (3.3) is elementary). The second inequality holds because $d_{14}$ is positive imaginary and $d_{32}$ is negative imaginary. Finally, $d_{13} \neq 0$ because $\operatorname{Im} a_{1}>0$ and $\operatorname{Im} a_{3}<0$. We may now conclude, if $x$ and $y$ are finite, that

$$
U_{14}>U_{13}>0 \text { and }-U_{13}<U_{12}<U_{13} \text {. }
$$

By (3.4) it follows that $M>0, L_{+}>L_{-}>0$, and $L_{-}^{2}-M^{2}>0$, whence

$$
L_{+}>L_{-}>M>0 \text {. }
$$

Since $L_{ \pm}^{2}-M^{2}$ depends only on the quantities listed in (3.7), which are independent of $x$ and $y$, both (3.9) and (3.8) are still valid if either $x$ or $y$ is infinite, but not both. If the interval of integration is the whole real line, then $U_{14}=U_{13}=-U_{12}=+\infty$ and $M=0$, as we shall show later.

Again assuming $x$ and $y$ to be finite, we shall now express $M$ and $L_{ \pm}$in terms of $f_{i}, g_{i}$, and $h_{i}$. Let

$$
\begin{array}{ll}
\xi_{1}=X_{1} X_{4}=\left(f_{1}+g_{1} x+h_{1} x^{2}\right)^{1 / 2}, & \xi_{2}=X_{2} X_{3}=\left(f_{2}+g_{2} x+h_{2} x^{2}\right)^{1 / 2}, \\
\eta_{1}=Y_{1} Y_{4}=\left(f_{1}+g_{1} y+h_{1} y^{2}\right)^{1 / 2}, & \eta_{2}=Y_{2} Y_{3}=\left(f_{2}+g_{2} y+h_{2} y^{2}\right)^{1 / 2}, \\
\zeta_{1}=X_{1} Y_{4}+Y_{1} X_{4}=2 \operatorname{Re}\left(X_{1} Y_{4}\right), & \zeta_{2}=X_{2} Y_{3}+Y_{2} X_{3}=2 \operatorname{Re}\left(X_{2} Y_{3}\right), \\
\theta_{1}=X_{1}^{2} Y_{4}^{2}+Y_{1}^{2} X_{4}^{2}=\zeta_{1}^{2}-2 \xi_{1} \eta_{1}, & \theta_{2}=X_{2}^{2} Y_{3}^{2}+Y_{2}^{2} X_{3}^{2}=\zeta_{2}^{2}-2 \xi_{2} \eta_{2} .
\end{array}
$$

Then $\xi_{i}, \eta_{i}$, and $\zeta_{i}$ are positive, but $\theta_{i}$ need not be. By (3.4) and (3.5) we see that

$$
M=\zeta_{1} \zeta_{2} /(x-y), \quad \zeta_{i}^{2}=\left(\xi_{i}+\eta_{i}\right)^{2}-h_{i}(x-y)^{2},
$$

where the second equation follows from

$$
\begin{aligned}
\zeta_{1}^{2} & =\left(X_{1} Y_{4}+Y_{1} X_{4}\right)^{2}=\left(X_{1} X_{4}+Y_{1} Y_{4}\right)^{2}-\left(X_{1}^{2}-Y_{1}^{2}\right)\left(X_{4}^{2}-Y_{4}^{2}\right) \\
& =\left(\xi_{1}+\eta_{1}\right)^{2}-h_{1}(x-y)^{2}
\end{aligned}
$$

and similarly for $\zeta_{2}^{2}$.

If we define

$$
\delta_{i j}=\left(2 f_{i} h_{j}+2 f_{j} h_{i}-g_{i} g_{j}\right)^{1 / 2},
$$

then $\delta_{i i}>0$ because $f_{i}+g_{i} t+h_{i} t^{2}>0$ for all real $t$. A stronger result than $\delta_{12}>0$ will be given in (3.17). By (3.2) we have

$$
a_{1} b_{4}+a_{4} b_{1}=g_{1}, \quad\left(a_{1} b_{4}\right)\left(a_{4} b_{1}\right)=f_{1} h_{1} .
$$


We solve these two equations for $a_{1} b_{4}$ and use the assumption $\operatorname{Im}\left(a_{1}\right)>0$ to find

$$
a_{1} b_{4}=\left(g_{1}+i \delta_{11}\right) / 2, \quad d_{14}=a_{1} b_{4}-a_{4} b_{1}=i \delta_{11} .
$$

A similar procedure yields

$$
a_{2} b_{3}=\left(g_{2}+i \delta_{22}\right) / 2, \quad d_{23}=i \delta_{22} .
$$

Thus we find

$$
\begin{aligned}
\left|d_{12}^{2}\right| & =d_{12} d_{43}=\left(a_{1} b_{2}-a_{2} b_{1}\right)\left(a_{4} b_{3}-a_{3} b_{4}\right) \\
& =f_{1} h_{2}+f_{2} h_{1}-2 \operatorname{Re}\left[\left(g_{1}+i \delta_{11}\right)\left(g_{2}-i \delta_{22}\right) / 4\right] \\
& =\left(\delta_{12}^{2}-\delta_{11} \delta_{22}\right) / 2 .
\end{aligned}
$$

Since $d_{12} \neq 0$, except in the excluded degenerate case (cf. (3.7)), we have

$$
\delta_{12}^{2}>\delta_{11} \delta_{22}>0 \text {. }
$$

A similar calculation leads to

$$
\left|d_{13}^{2}\right|=\left(\delta_{12}^{2}+\delta_{11} \delta_{22}\right) / 2 .
$$

We define

$$
\Delta=\left(\delta_{12}^{4}-\delta_{11}^{2} \delta_{22}^{2}\right)^{1 / 2}, \quad \Delta_{ \pm}=\delta_{12}^{2} \pm \Delta>0,
$$

and use (3.7) and the last equation of (3.4) to get

$$
\begin{array}{lll}
U_{14}^{2}-U_{12}^{2}=\left(\delta_{12}^{2}+\delta_{11} \delta_{22}\right) / 2, & & U_{14}^{2}-U_{13}^{2}=\left(\delta_{12}^{2}-\delta_{11} \delta_{22}\right) / 2, \\
U_{13}^{2}-U_{12}^{2}=\delta_{11} \delta_{22}, & & L_{ \pm}^{2}-M^{2}=\Delta_{ \pm} .
\end{array}
$$

The last equation and (3.11) allow calculation of $M^{2}$ and $L_{ \pm}^{2}$.

If the interval of integration is infinite, we take the appropriate limit in (3.11) to find (2.31), (2.33), or (2.34). In the first two cases, $M^{2}$ is a product of two factors such as

$$
2 h_{i}^{1 / 2} \xi_{i}-g_{i}-2 h_{i} x=\left[\left(g_{i}+2 h_{i} x\right)^{2}+\delta_{i i}^{2}\right]^{1 / 2}-\left(g_{i}+2 h_{i} x\right)>0 .
$$

Hence, $M>0$, except when the interval of integration is the whole real line.

An integral of the second kind used in previous parts of this table [5, $(2.14)$, (2.17)] is

$$
\begin{aligned}
I_{2} & =[1,-1,-1,-3] \\
& =2 d_{12} d_{13} R_{D}\left(U_{12}^{2}, U_{13}^{2}, U_{14}^{2}\right) / 3+2 X_{1} Y_{1} / X_{4} Y_{4} U_{14} .
\end{aligned}
$$

(Since $p_{1} \neq p_{4}$, this integral is now complex.) Putting $w=z$ in [7, (8.5), (5.5)] to obtain the Landen transformation of $R_{D}$, and using the notation in (3.4), we find

$$
\begin{aligned}
\Delta R_{D}\left(U_{12}^{2}, U_{13}^{2}, U_{14}^{2}\right)= & 8 \Delta_{+} R_{D}\left(M^{2}, L_{-}^{2}, L_{+}^{2}\right) \\
& -12 R_{F}\left(M^{2}, L_{-}^{2}, L_{+}^{2}\right)+6 / U_{14} .
\end{aligned}
$$

Substituting in (3.21) and using the identities

$$
\begin{aligned}
& d_{12} d_{13} d_{24} d_{34}=\left|d_{12} d_{13}\right|^{2}=\Delta^{2} / 4, \\
& 4 d_{24} d_{34} X_{1}^{2} Y_{1}^{2}=\delta_{12}^{2} \theta_{1}-\delta_{11}^{2} \theta_{2}-i 2 \delta_{11} \xi_{1} \eta_{1} U_{14} A(-1,1,1,-1),
\end{aligned}
$$


we get

$$
I_{2}=4 d_{12} d_{13}\left[2 G-2 \Delta R_{F}-i \delta_{11} A(-1,1,1,-1)\right] / \Delta^{2},
$$

(3.25) $G=2 \Delta \Delta_{+} R_{D}\left(M^{2}, L_{-}^{2}, L_{+}^{2}\right) / 3+\Delta / 2 U+\left(\delta_{12}^{2} \theta_{1}-\delta_{11}^{2} \theta_{2}\right) / 4 \xi_{1} \eta_{1} U$,

where we denote $R_{F}\left(M^{2}, L_{-}^{2}, L_{+}^{2}\right)$ by $R_{F}$ and $U_{14}$ by $U$ for brevity.

The integrals $[-3,1,1,-3]$ and $[-3,-1,-1,-3]$ can now be obtained from $[8,(2.24),(2.23)]$. In the second case, we use the identity

$$
\begin{aligned}
2 d_{12} d_{13} & =\left[h_{1} \delta_{12}^{2}-h_{2} \delta_{11}^{2}+i\left(g_{1} h_{2}-g_{2} h_{1}\right) \delta_{11}\right] / h_{1} \\
& =\delta_{12}^{2}-\Lambda_{0}+i \psi_{0} \delta_{11} / h_{1} .
\end{aligned}
$$

\section{INTEGRALS OF THE THIRD KIND}

We shall encounter $R_{J}\left(U_{12}^{2}, U_{13}^{2}, U_{14}^{2}, W^{2}\right)$, where the first three variables are real but $W^{2}$ is complex. The function can be changed by Landen transformation [8, (4.14)] into $R_{J}\left(M^{2}, L_{-}^{2}, L_{+}^{2}, W_{+}^{2}\right)$, but $W_{+}^{2}$ also is complex. Instead, an inverse Landen transformation followed by the duplication theorem leads to $R_{J}\left(M^{2}, L_{-}^{2}, L_{+}^{2}, \Omega^{2}\right)$ with real $\Omega^{2}$. This combination of two transformations (see Appendix A) is equivalent to a direct Landen transformation for integrals of the first and second kinds but not the third kind.

In (A.8) we identify $\left(z_{-}, z_{+}, \alpha\right)$ with $\left(U_{12}, U_{13}, U_{14}\right)$ and find from (A.9) and (3.4) that $\left(x^{2}+\lambda, y^{2}+\lambda, z^{2}+\lambda\right)=\left(L_{-}^{2}, L_{+}^{2}, M^{2}\right)$. Because of [5, (2.15), (2.9)], we put

$$
w_{+}^{2}=W^{2}=U_{14}^{2}-d_{12} d_{13} d_{45} / d_{15} .
$$

Since $d_{15}$ and $d_{45}$ are complex conjugates, it follows that $\left|\alpha^{2}-w_{+}^{2}\right|^{2}=\left|d_{12} d_{13}\right|^{2}$. By (3.7) the condition (A.2) is satisfied, and so $w_{-}^{2}$ is the complex conjugate of $w_{+}^{2}$ :

$$
w_{-}^{2}=U_{14}^{2}-d_{43} d_{42} d_{15} / d_{45}
$$

We define

$$
\Omega^{2}=w^{2}+\lambda, \quad w=w_{+} w_{-} / U_{14},
$$

and find from (A.3), (3.7), and [4, (5.22)] that

$$
\begin{aligned}
\Omega^{2}-M^{2} & =w^{2}-z^{2}=w_{+}^{2}+w_{-}^{2}-z_{+}^{2}-z_{-}^{2} \\
& =\left(U_{14}^{2}-U_{13}^{2}\right)+\left(U_{14}^{2}-U_{12}^{2}\right)-d_{12} d_{13} d_{45} / d_{15}-d_{43} d_{42} d_{15} / d_{45} \\
& =\left(d_{12} d_{45}-d_{42} d_{15}\right)\left(d_{43} d_{15}-d_{13} d_{45}\right) / d_{15} d_{45} \\
& =\left|\left(d_{12} d_{45}-d_{42} d_{15}\right) / d_{15}\right|^{2}=\left|d_{14} d_{25} / d_{15}\right|^{2} .
\end{aligned}
$$

Defining

$$
\gamma_{i}=\left|d_{i 5}\right|^{2}=f_{i} b_{5}^{2}-g_{i} a_{5} b_{5}+h_{i} a_{5}^{2}, \quad i=1,2,
$$

we note that $\gamma_{i}>0$ because $\operatorname{Im} d_{i 5} \neq 0$. By (3.14) we have

$$
\Omega^{2}=M^{2}+\Lambda, \quad w^{2}=z^{2}+\Lambda, \quad \Lambda=\delta_{11}^{2} \gamma_{2} / \gamma_{1}>0 .
$$

Since $\Omega^{2}>0$, we may choose $\Omega>0$. Then it follows from (3.9), (A.5), (4.3), and (3.20) that

$$
L_{+}>\Omega>L_{-}>M \geq 0, \quad \Delta_{+}>\Lambda>\Delta_{-}>0,
$$


where $M=0$ by (2.34) only if the interval of integration is the whole real line. It will be useful to define also (for $i=1,2$ )

$$
\alpha_{i 5}=\partial \gamma_{i} / \partial b_{5}=2 f_{i} b_{5}-g_{i} a_{5}, \quad \beta_{i 5}=-\partial \gamma_{i} / \partial a_{5}=g_{i} b_{5}-2 h_{i} a_{5},
$$

whence

$$
\gamma_{i}=\left(\alpha_{i 5} b_{5}-\beta_{i 5} a_{5}\right) / 2 \text {. }
$$

(The definitions (4.7) are equivalent to $\alpha_{15}=a_{1} d_{45}+a_{4} d_{15}$ and $\beta_{15}=b_{1} d_{45}+$ $b_{4} d_{15}$, and similar relations for $\alpha_{25}$ and $\beta_{25}$.)

From (4.1) and (4.2) we can obtain a coefficient in (A.8):

$$
\begin{aligned}
w_{+}^{2}-w_{-}^{2} & =-d_{12} d_{13} d_{45} / d_{15}+d_{43} d_{42} d_{15} / d_{45} \\
& =-\left(2 i / \gamma_{1}\right) \operatorname{Im}\left(d_{12} d_{13} d_{45}^{2}\right) .
\end{aligned}
$$

It is straightforward to show by (3.6) and (3.2) that

$$
d_{12} d_{45}=a_{5}\left(h_{1} a_{2}-a_{1} b_{4} b_{2}\right)+b_{5}\left(f_{1} b_{2}-a_{4} b_{1} a_{2}\right) .
$$

Replacing the subscript 2 by 3 , multiplying $d_{12} d_{45}$ by $d_{13} d_{45}$, and taking the imaginary part with the help of (3.14), we find

$$
w_{+}^{2}-w_{-}^{2}=-i \delta_{11} \psi / \gamma_{1},
$$

where

$$
\begin{aligned}
\psi & =\left(\alpha_{15} \beta_{25}-\alpha_{25} \beta_{15}\right) / 2 \\
& =a_{5}^{2}\left(g_{1} h_{2}-g_{2} h_{1}\right)-2 a_{5} b_{5}\left(f_{1} h_{2}-f_{2} h_{1}\right)+b_{5}^{2}\left(f_{1} g_{2}-f_{2} g_{1}\right) .
\end{aligned}
$$

Incidentally, with the help of (A.3) and (3.19) we see that

$$
\begin{aligned}
\left(w_{+}^{2}-w_{-}^{2}\right)^{2} & =\left(x^{2}-w^{2}\right)\left(y^{2}-w^{2}\right)=\left(L_{-}^{2}-\Omega^{2}\right)\left(L_{+}^{2}-\Omega^{2}\right) \\
& =\left(\Delta_{-}-\Lambda\right)\left(\Delta_{+}-\Lambda\right)=\Lambda^{2}-2 \delta_{12}^{2} \Lambda+\delta_{11}^{2} \delta_{22}^{2} .
\end{aligned}
$$

Substituting $\Lambda$ from (4.5) and comparing with (4.9), we get

$$
0>\left(w_{+}^{2}-w_{-}^{2}\right)^{2}=-\Lambda \psi^{2} / \gamma_{1} \gamma_{2},
$$

where

$$
\psi^{2}=-\delta_{11}^{2} \gamma_{2}^{2}+2 \delta_{12}^{2} \gamma_{1} \gamma_{2}-\delta_{22}^{2} \gamma_{1}^{2}>0
$$

a result that is tedious to obtain by squaring $\psi$. Although it provides a useful numerical check, the last equation does not determine the sign of $\psi$, which may be positive or negative.

Since $b=w\left(w^{2}+\lambda\right)=w \Omega^{2}$ by (A.6), we can now write (A.8) as

$$
\begin{aligned}
2\left(W^{2}\right. & \left.-U_{14}^{2}\right) R_{J}\left(U_{12}^{2}, U_{13}^{2}, U_{14}^{2}, W^{2}\right) \\
= & -i\left(\delta_{11} \psi / \gamma_{1}\right)\left[2 R_{J}\left(M^{2}, L_{-}^{2}, L_{+}^{2}, \Omega^{2}\right)+3 R_{C}\left(a^{2}, b^{2}\right)\right] \\
& +6 R_{F}\left(M^{2}, L_{-}^{2}, L_{+}^{2}\right)-3 R_{C}\left(z^{2}, w^{2}\right),
\end{aligned}
$$

where $W^{2}$ is given in (4.1) and

$$
\begin{aligned}
& z=U_{12} U_{13} / U_{14}, \quad w^{2}-z^{2}=\Omega^{2}-M^{2}=\Lambda=\delta_{11}^{2} \gamma_{2} / \gamma_{1}, \\
& b=w \Omega^{2}, \quad a^{2}-b^{2}=\Lambda^{2} \psi^{2} / \gamma_{1} \gamma_{2}=\Lambda\left(\Delta_{+}-\Lambda\right)\left(\Lambda-\Delta_{-}\right) .
\end{aligned}
$$


An integral of the third kind used in previous parts of this table [5, (2.15), (2.17)] is

$$
\begin{aligned}
I_{3} & =[1,-1,-1,-1,-2] \\
& =2 d_{12} d_{13} d_{14} R_{J}\left(U_{12}^{2}, U_{13}^{2}, U_{14}^{2}, W^{2}\right) / 3 d_{15}+2 R_{C}\left(P^{2}, Q^{2}\right) .
\end{aligned}
$$

Substituting (4.12) and using (4.1), (3.14), and (4.4), we find

$$
\begin{aligned}
I_{3} / 2 d_{15}= & -\delta_{11}^{2} \psi\left[R_{J}\left(M^{2}, L_{-}^{2}, L_{+}^{2}, \Omega^{2}\right) / 3+R_{C}\left(a^{2}, b^{2}\right) / 2\right] / \gamma_{1}^{2} \\
& -i \delta_{11} R_{F} / \gamma_{1}+i \delta_{11} R_{C}\left(z^{2}, w^{2}\right) / 2 \gamma_{1}+R_{C}\left(P^{2}, Q^{2}\right) / d_{15},
\end{aligned}
$$

where $R_{F}=R_{F}\left(M^{2}, L_{-}^{2}, L_{+}^{2}\right)$.

We shall separate the real and imaginary parts of the last term,

$$
R_{C}\left(P^{2}, Q^{2}\right) / d_{15}=R_{C}\left(\left(d_{15} P\right)^{2},\left(d_{15} Q\right)^{2}\right),
$$

and find that the imaginary part cancels the next to last term. Putting $d_{15} P=$ $X+i Y$ and referring to (B.1) in Appendix B, we shall need $X, Y, X^{2}+$ $Y^{2},\left|d_{15} Q\right|^{4}$, and

$$
c=d_{15}^{2}\left(Q^{2}-P^{2}\right)=-d_{25} d_{35} d_{15} d_{45}=-\gamma_{1} \gamma_{2},
$$

where we have used $[5,(2.5)]$ and (4.4). It follows from [5, (2.8)] and (3.10) that

$$
(x-y)(X+i Y)=(x-y) d_{15} P=\left(\eta_{5} \xi_{2} / \xi_{1}\right) d_{15} X_{4}^{2}+\left(\xi_{5} \eta_{2} / \eta_{1}\right) d_{15} Y_{4}^{2},
$$

where

$$
\xi_{5}=X_{5}^{2}=a_{5}+b_{5} x, \quad \eta_{5}=Y_{5}^{2}=a_{5}+b_{5} y .
$$

Using (3.14) and (4.7), we find

$$
2 d_{15} X_{4}^{2}=2\left(a_{1} b_{5}-a_{5} b_{1}\right)\left(a_{4}+b_{4} x\right)=\alpha_{15}+\beta_{15} x+i \delta_{11} \xi_{5},
$$

and similarly for $d_{15} Y_{4}^{2}$. Substitution in (4.18) yields

$$
\begin{aligned}
& 2(x-y) X=\eta_{5}\left(\alpha_{15}+\beta_{15} x\right) \xi_{2} / \xi_{1}+\xi_{5}\left(\alpha_{15}+\beta_{15} y\right) \eta_{2} / \eta_{1}, \\
& 2 Y=\delta_{11} \xi_{5} \eta_{5}\left(\xi_{2} / \xi_{1}+\eta_{2} / \eta_{1}\right) /(x-y)=\delta_{11} \xi_{5} \eta_{5} U / \xi_{1} \eta_{1} .
\end{aligned}
$$

Instead of squaring $X$ and $Y$, it is easier to get $X^{2}+Y^{2}$ by calculating $\left|d_{15} P\right|^{2}=\gamma_{1}|P|^{2}$ from [5, (2.8)]:

$$
\begin{aligned}
(x-y)^{2}|P|^{2} & =\left(\xi_{5} \eta_{2}\right)^{2}+\left(\eta_{5} \xi_{2}\right)^{2}+\xi_{5} \eta_{5} \xi_{2} \eta_{2}\left(X_{4} Y_{1} / X_{1} Y_{4}+X_{1} Y_{4} / X_{4} Y_{1}\right) \\
& =\left(\xi_{5} \eta_{2}\right)^{2}+\left(\eta_{5} \xi_{2}\right)^{2}+\xi_{5} \eta_{5} \theta_{1} \xi_{2} \eta_{2} / \xi_{1} \eta_{1} .
\end{aligned}
$$

From $\gamma_{2}=d_{25} d_{35}$ and $(x-y) d_{i 5}=\xi_{5} Y_{i}^{2}-\eta_{5} X_{i}^{2}$ it follows by (3.10) that

$$
(x-y)^{2} \gamma_{2}=\left(\xi_{5} \eta_{2}\right)^{2}+\left(\eta_{5} \xi_{2}\right)^{2}-\xi_{5} \eta_{5} \theta_{2},
$$

and hence

$$
(x-y)^{2}|P|^{2}=\xi_{5} \eta_{5}\left(\theta_{2}+\theta_{1} \xi_{2} \eta_{2} / \xi_{1} \eta_{1}\right)+(x-y)^{2} \gamma_{2} .
$$

Defining

$$
S=U z=U_{12} U_{13}
$$


we find

$$
\begin{aligned}
(x-y)^{2} S & =\left(X_{1} X_{2} Y_{3} Y_{4}+Y_{1} Y_{2} X_{3} X_{4}\right)\left(X_{1} X_{3} Y_{2} Y_{4}+Y_{1} Y_{3} X_{2} X_{4}\right) \\
& =\xi_{1} \eta_{1}\left(X_{2}^{2} Y_{3}^{2}+Y_{2}^{2} X_{3}^{2}\right)+\xi_{2} \eta_{2}\left(X_{1}^{2} Y_{4}^{2}+Y_{1}^{2} X_{4}^{2}\right) \\
& =\xi_{1} \eta_{1} \theta_{2}+\xi_{2} \eta_{2} \theta_{1}
\end{aligned}
$$

and thus

$$
\begin{aligned}
& |P|^{2}=\xi_{5} \eta_{5} S / \xi_{1} \eta_{1}+\gamma_{2}, \\
& X^{2}+Y^{2}=\gamma_{1} \xi_{5} \eta_{5} S / \xi_{1} \eta_{1}+\gamma_{1} \gamma_{2}=\mu S+\gamma_{1} \gamma_{2}=T-\gamma_{1} \gamma_{2}, \\
& \mu=\gamma_{1} \xi_{5} \eta_{5} / \xi_{1} \eta_{1}, \quad T=\mu S+2 \gamma_{1} \gamma_{2} .
\end{aligned}
$$

Finally, [5, (2.5)], (4.1), and (4.3) imply

$$
\begin{aligned}
\left|d_{15} Q\right|^{4}=\left(\mu w_{+} w_{-}\right)^{2} & =(\mu U w)^{2}=\mu^{2} U^{2}\left(z^{2}+\Lambda\right)=V^{2}, \\
V^{2} & =\mu^{2}\left(S^{2}+U^{2} \Lambda\right)
\end{aligned}
$$

while

$$
X^{2}+Y^{2}+c=\mu S+\gamma_{1} \gamma_{2}-\gamma_{1} \gamma_{2}=\mu U z
$$

From (B.1) we now have

$$
\begin{aligned}
R_{C}\left(P^{2}, Q^{2}\right) / d_{15} & \\
\quad & \left.=X R_{C}\left(T^{2}, V^{2}\right)-i\left(\delta_{11} \mu U / 2 \gamma_{1}\right) R_{C}\left((\mu U z)^{2},(\mu U w)^{2}\right)\right) \\
& =X R_{C}\left(T^{2}, V^{2}\right)-i\left(\delta_{11} / 2 \gamma_{1}\right) R_{C}\left(z^{2}, w^{2}\right) .
\end{aligned}
$$

The last term cancels a term in (4.15) to yield

$$
I_{3}=-2 d_{15}\left(H+i \delta_{11} R_{F} / \gamma_{1}\right),
$$

where

$$
H=\delta_{11}^{2} \psi\left[R_{J}\left(M^{2}, L_{-}^{2}, L_{+}^{2}, \Omega^{2}\right) / 3+R_{C}\left(a^{2}, b^{2}\right) / 2\right] / \gamma_{1}^{2}-X R_{C}\left(T^{2}, V^{2}\right) .
$$

Using a subscript 0 to label quantities in which we have put $a_{5}=1$ and $b_{5}=0$, we obtain from $[5,(2.17)]$ also

$$
I_{3}^{\prime}=[1,-1,-1,-1]=2 b_{1}\left(H_{0}+i \delta_{11} R_{F} / h_{1}\right),
$$

where

$$
H_{0}=\delta_{11}^{2} \psi_{0}\left[R_{J}\left(M^{2}, L_{-}^{2}, L_{+}^{2}, \Omega_{0}^{2}\right) / 3+R_{C}\left(a_{0}^{2}, b_{0}^{2}\right) / 2\right] / h_{1}^{2}-X_{0} R_{C}\left(T_{0}^{2}, V_{0}^{2}\right) .
$$

Since $I_{1}, I_{2}, I_{3}$, and $I_{3}^{\prime}$ have now been reduced to $R$-functions of real variables, the ten integrals of the third kind in $\S 2$ can be derived by substitution in the formulas of $[5,8]$ (the latter if the odd $p$ 's are not in decreasing order). Converting coefficients to the notation of this paper is straightforward but sometimes tedious. In addition to the recurrence relation [5, (4.8)], the following identities are useful:

$$
\begin{gathered}
2 d_{24} d_{34} X_{1}^{2} Y_{1}^{2}=\left(\delta_{12}^{2} \theta_{1}-\delta_{11}^{2} \theta_{2}\right) / 2-i \delta_{11} \xi_{1} \eta_{1} U A(-1,1,1,-1), \\
h_{1}^{1 / 2} A(1,1,1,-1)=B+i \delta_{11} A(-1,1,1,-1) / 2, \\
h_{1}^{1 / 2} A(3,1,1,1)=E+i \delta_{11} A(1,1,1,1) / 2,
\end{gathered}
$$

where $B$ and $E$ are defined in (2.3). 


\section{NUMERICAL CHECKS}

The 13 integrals in $\S 2$ were checked numerically when $x=2, y=-3$, $\left(f_{1}, g_{1}, h_{1}\right)=(2.7,-1.8,0.9),\left(f_{2}, g_{2}, h_{2}\right)=(2.0,2.4,0.8)$, and $\left(a_{5}, b_{5}\right)=$ $(1.1,-0.4)$. (The zeros of the quadratic polynomials are $1 \pm i \sqrt{2}$ and $(-3 \pm i) / 2$. Because $S<0$, the validity condition [3, (33)] is violated (cf. (4.25)), and the integral of the first kind would have to be split in two parts before using [3, (34)].) In each of the formulas (2.36) to (2.48) the integral on the left side, defined by (2.35), was integrated numerically by the SLATEC code QNG. On the right side the quantities $R_{F}, G, H, H_{0}$ were calculated by using the codes for $R$-functions in the Supplements to [4, 5], and the remaining calculations were done with a hand calculator. For each of the 13 cases the values obtained for the two sides agreed to better than one part in a million (better than the claimed accuracy of QNG).

Some intermediate values are

$$
\begin{array}{ll}
M^{2}=0.36362947, & R_{F}\left(M^{2}, L_{-}^{2}, L_{+}^{2}\right)=0.54784092, \\
L_{-}^{2}=0.53423014, & R_{D}\left(M^{2}, L_{-}^{2}, L_{+}^{2}\right)=0.042910488, \\
L_{+}^{2}=24.673029, & R_{J}\left(M^{2}, L_{-}^{2}, L_{+}^{2}, \Omega^{2}\right)=0.048599080, \\
\Omega^{2}=21.199185, & R_{J}\left(M^{2}, L_{-}^{2}, L_{+}^{2}, \Omega_{0}^{2}\right)=0.12739513, \\
\Omega_{0}^{2}=6.1236295, & R_{C}\left(a^{2}, b^{2}\right)=0.0098889795, \\
a^{2}=11237.193, & R_{C}\left(a_{0}^{2}, b_{0}^{2}\right)=0.050085175, \\
b^{2}=9741.4746, & R_{C}\left(T^{2}, V^{2}\right)=0.58372845, \\
a_{0}^{2}=844.71933, & R_{C}\left(T_{0}^{2}, V_{0}^{2}\right)=0.94657139, \\
b_{0}^{2}=247.52253, & G=10.495586, \\
T^{2}=10.288757, & H=0.049905556, \\
V^{2}=1.1362990, & H_{0}=-1.8557835, \\
T_{0}^{2}=1.1328716, & A(-1,1,1,-1)=1.5731367, \\
V_{0}^{2}=1.1077327, & A(1,1,1,1)=-0.49594737, \\
X=-1.1571677, & A(-1,1,1,-1,-2)=6.2622360 \\
X_{0}=-0.093427949, & A(1,1,1,1,-2)=14.845682 .
\end{array}
$$

As a test of Cauchy principal values, the three integrals with $p_{5}=-2$, viz. (2.39), (2.46), and (2.47), were checked numerically with the same values of $x, y, f_{i}, g_{i}$, and $h_{i}$ as before but with $a_{5}+b_{5} t=t$, so that each integrand has a simple pole in the open interval of integration. In each case the Cauchy principal value of the left side was computed by the SLATEC code QAWC, and the right side was calculated as before. Cauchy principal values are not required for either $R_{J}$ or $R_{C}$, as one can see from (4.6) and (2.19), since $V^{2}>0$ whether $\xi_{5} \eta_{5}$ is positive or negative. For each of the three cases the values obtained for the two sides agreed to better than one part in a million, even though the SLATEC code issued a warning about impairment of accuracy by roundoff error in the case of (2.47). 


\section{APPENDIX A. $R_{J}$ WITH A RESTRICTED COMPLEX PARAMETER}

When the fourth variable of $R_{J}$ is complex but has a special relation (see (A.2)) to the first three variables, which are real, transformation (A.8) leads to $R_{J}$ with four real variables. To derive this, we start from the inverse Landen transformation [7, (8.5), (5.7), (7.2)],

$$
\begin{aligned}
2\left(w_{+}^{2}-\alpha^{2}\right) R_{J}\left(z_{-}^{2}, z_{+}^{2}, \alpha^{2}, w_{+}^{2}\right)= & \left(w_{+}^{2}-w_{-}^{2}\right) R_{J}\left(x^{2}, y^{2}, z^{2}, w^{2}\right) \\
& +3 R_{F}\left(x^{2}, y^{2}, z^{2}\right)-3 R_{C}\left(z^{2}, w^{2}\right), \\
y+x=2 \alpha, \quad y-x=(2 / \alpha)\left[\left(\alpha^{2}-z_{+}^{2}\right)\left(\alpha^{2}-z_{-}^{2}\right)\right]^{1 / 2}, \quad z=z_{+} z_{-} / \alpha, & \left(\alpha^{2}-w_{+}^{2}\right)\left(\alpha^{2}-w_{-}^{2}\right)=\left(\alpha^{2}-z_{+}^{2}\right)\left(\alpha^{2}-z_{-}^{2}\right) .
\end{aligned}
$$

We are concerned with the case in which $w_{+}^{2}$ is not real, $\alpha>z_{+}>0$, and $-z_{+} \leq z_{-} \leq z_{+}$. (We exclude the degenerate case $\alpha=z_{+}$, in which $R_{J}$ is elementary, $w_{-}^{2}=\alpha^{2}$, and $w^{2}=w_{+}^{2}$, whence $w^{2}$ is complex.) The last equation in (A.1) defines $w_{-}^{2}$ and shows, since the right side is positive, that $\alpha^{2}-w_{+}^{2}$ and $\alpha^{2}-w_{-}^{2}$ have equal and opposite complex phases. If they have also the same absolute value, i.e., if

$$
\left|\alpha^{2}-w_{+}^{2}\right|^{2}=\left(\alpha^{2}-z_{+}^{2}\right)\left(\alpha^{2}-z_{-}^{2}\right),
$$

then $w_{-}^{2}$ is the complex conjugate of $w_{+}^{2}$, and hence $w^{2}>0$. Since $w$ cannot vanish, we may choose $w>0$, whence $w_{-}$is the complex conjugate of $w_{+}$.

From (A.1) and (A.2) we see that $y>x>0$ and

$$
\begin{array}{ll}
x y+z^{2}=z_{+}^{2}+z_{-}^{2}, & x y+w^{2}=w_{+}^{2}+w_{-}^{2}, \\
(x+y) z=2 z_{+} z_{-}, & (x+y) w=2 w_{+} w_{-}, \\
(x \pm z)(y \pm z)=\left(z_{+} \pm z_{-}\right)^{2}, & (x \pm w)(y \pm w)=\left(w_{+} \pm w_{-}\right)^{2}, \\
w^{2}-z^{2}=w_{+}^{2}+w_{-}^{2}-z_{+}^{2}-z_{-}^{2} . &
\end{array}
$$

We find also that

$$
\begin{aligned}
& x^{2}-z^{2}=\left[\left(\alpha^{2}-z_{-}^{2}\right)^{1 / 2}-\left(\alpha^{2}-z_{+}^{2}\right)^{1 / 2}\right]^{2}, \\
& y^{2}-z^{2}=\left[\left(\alpha^{2}-z_{-}^{2}\right)^{1 / 2}+\left(\alpha^{2}-z_{+}^{2}\right)^{1 / 2}\right]^{2}, \\
& x^{2}-w^{2}=\left[\left(\alpha^{2}-w_{-}^{2}\right)^{1 / 2}-\left(\alpha^{2}-w_{+}^{2}\right)^{1 / 2}\right]^{2}, \\
& y^{2}-w^{2}=\left[\left(\alpha^{2}-w_{-}^{2}\right)^{1 / 2}+\left(\alpha^{2}-w_{+}^{2}\right)^{1 / 2}\right]^{2} .
\end{aligned}
$$

Since $(x-w)(y-w)=\left(w_{+}-w_{-}\right)^{2}<0$ and $x^{2}-z^{2} \geq 0$, we have

$$
y>w>x>0 \text { and }-x \leq z \leq x .
$$

If $z_{-}<0$, then $z<0$, and the $R$-functions in (A.1) do not take the principal values represented by (1.3) to (1.5). A remedy is provided by the duplication theorem $[7,(6.1)(8.7)]$ :

$$
\begin{aligned}
R_{F}\left(x^{2}, y^{2}, z^{2}\right)=2 R_{F} & \left(x^{2}+\lambda, y^{2}+\lambda, z^{2}+\lambda\right), \\
R_{J}\left(x^{2}, y^{2}, z^{2}, w^{2}\right)= & 2 R_{J}\left(x^{2}+\lambda, y^{2}+\lambda, z^{2}+\lambda, w^{2}+\lambda\right) \\
& +3 R_{C}\left(a^{2}, b^{2}\right),
\end{aligned}
$$


with

$$
\begin{gathered}
\lambda=x y+x z+y z, \quad a=w^{2}(x+y+z)+x y z, \\
b=w\left(w^{2}+\lambda\right), \quad b \pm a=(w \pm x)(w \pm y)(w \pm z) .
\end{gathered}
$$

It follows from (A.5) that $b-a<0$. Since $z^{2}+\lambda=(z+x)(z+y)$ is a product of nonnegative factors, and $w^{2}+\lambda>z^{2}+\lambda$ by (A.5), we have

$$
a>b>0 \text {. }
$$

Finally, we combine (A.6) and (A.1):

Theorem. If (A.2) holds, let $w_{-}$be the complex conjugate of $w_{+}$. Then

$$
\begin{aligned}
2\left(w_{+}^{2}\right. & \left.-\alpha^{2}\right) R_{J}\left(z_{-}^{2}, z_{+}^{2}, \alpha^{2}, w_{+}^{2}\right) \\
= & \left(w_{+}^{2}-w_{-}^{2}\right)\left[2 R_{J}\left(x^{2}+\lambda, y^{2}+\lambda, z^{2}+\lambda, w^{2}+\lambda\right)+3 R_{C}\left(a^{2}, b^{2}\right)\right] \\
& +6 R_{F}\left(x^{2}+\lambda, y^{2}+\lambda, z^{2}+\lambda\right)-3 R_{C}\left(z^{2}, w^{2}\right)
\end{aligned}
$$

where

$$
\begin{aligned}
& \alpha>z_{+}>0, \quad-z_{+} \leq z_{-} \leq z_{+}, \quad \operatorname{Im}\left(w_{+}^{2}\right) \neq 0, \\
& z=z_{+} z_{-} / \alpha, \quad w=w_{+} w_{-} / \alpha, \quad z^{2}+\lambda=\left(z_{+}+z_{-}\right)^{2}, \\
& x^{2}+\lambda=\left(z_{+}+z_{-}\right)^{2}+\left[\left(\alpha^{2}-z_{-}^{2}\right)^{1 / 2}-\left(\alpha^{2}-z_{+}^{2}\right)^{1 / 2}\right]^{2}, \\
& y^{2}+\lambda=\left(z_{+}+z_{-}\right)^{2}+\left[\left(\alpha^{2}-z_{-}^{2}\right)^{1 / 2}+\left(\alpha^{2}-z_{+}^{2}\right)^{1 / 2}\right]^{2}, \\
& w^{2}+\lambda=w_{+}^{2}+w_{-}^{2}+2 z_{+} z_{-}, \quad a=\left[\left(w_{+}^{2}+w_{-}^{2}\right) z_{+} z_{-}+2 w_{+}^{2} w_{-}^{2}\right] / \alpha, \\
& b=w_{+} w_{-}\left(w_{+}^{2}+w_{-}^{2}+2 z_{+} z_{-}\right) / \alpha, \\
& b \pm a=\left(w_{+} \pm w_{-}\right)^{2}\left(w_{+} w_{-} \pm z_{+} z_{-}\right) / \alpha, \\
& b^{2}-a^{2}=\left(w_{+}^{2}-w_{-}^{2}\right)^{2}\left(w^{2}-z^{2}\right)=\left(w_{+}^{2}-w_{-}^{2}\right)^{2}\left(w_{+}^{2}+w_{-}^{2}-z_{+}^{2}-z_{-}^{2}\right) .
\end{aligned}
$$

Note that $z^{2}+\lambda$ is the square of a nonnegative quantity, even if $z<0$. The first term on the right side of (A.8) is pure imaginary while the second and third terms are real. If $z<0$, the third term is not represented by (1.5) until it is rewritten by the duplication theorem as $-6 R_{C}\left((z+w)^{2}, 2 w(z+w)\right)$; alternatively, it can be expressed in terms of an arctangent taken in the second quadrant rather than the fourth. Neither procedure is needed in this paper.

\section{APpendix B. Real AND IMAginary PARTS OF $R_{C}$}

In $\S 4$ we need to separate the real and imaginary parts of $R_{C}$ when its two variables are complex but differ by a real number. (If the difference is not real, it can be made real by using the homogeneity of $R_{C}$.)

Lemma. Let $x, y, c$ be real, $z=x+i y, r^{2}=x^{2}+y^{2}>0$, and $z^{2}+c \neq 0$. If $|c| \leq r^{2}$, then

$$
\begin{aligned}
R_{C}\left(z^{2}, z^{2}+c\right)= & x R_{C}\left(\left(r^{2}-c\right)^{2},\left|z^{2}+c\right|^{2}\right) \\
& -i y R_{C}\left(\left(r^{2}+c\right)^{2},\left|z^{2}+c\right|^{2}\right),
\end{aligned}
$$

where $R_{C}\left(z^{2}, z^{2}+c\right)$ denotes the branch that is continuous in $c$ and takes the value $1 / z$ when $c=0$. 
Proof. Let $|c|=a^{2} \leq r^{2}$. Then (B.1) reduces by [2, (6.9-15), (6.9-16)] to the correct equations

$$
\begin{gathered}
\log \frac{z+a}{z-a}=\log \left|\frac{z+a}{z-a}\right|-i \arctan \frac{2 a y}{r^{2}-a^{2}} \quad \text { if } c=-a^{2}, \\
\arctan \frac{a}{z}=\frac{1}{2} \arctan \frac{2 a x}{r^{2}-a^{2}}-\frac{i}{4} \log \frac{r^{2}+2 a y+a^{2}}{r^{2}-2 a y+a^{2}} \quad \text { if } c=a^{2} .
\end{gathered}
$$

On each right-hand side, the logarithm is taken real and the arctangent is taken in the first or fourth quadrant to get the principal value of the left side.

In the excluded case when $|c|>r^{2}$, the arctangent must be taken in the third or second quadrant, and the corresponding $R_{C}$ in (B.1) has the square of a negative number as its first argument. This can be replaced by the square of a positive number by using the duplication theorem [7, (3.7)], and $c$ can then have any real value provided $z^{2}+c \neq 0$. The result is given here although it is not needed in the present paper; it provides a way of computing $R_{C}$ with complex arguments.

Theorem. Let $x, y, c$ be real, $z=x+i y, r^{2}=x^{2}+y^{2}>0$, and $s=\left|z^{2}+c\right|>$ 0 . Then

$$
\begin{aligned}
& R_{C}\left(z^{2}, z^{2}+c\right)=x R_{C}\left(\sigma_{-}^{2}, s \sigma_{-}\right)-i y R_{C}\left(\sigma_{+}^{2}, s \sigma_{+}\right), \\
& s^{2}=\left|z^{2}+c\right|^{2}=\left(r^{2}-c\right)^{2}+4 c x^{2}=\left(r^{2}+c\right)^{2}-4 c y^{2}, \\
& \sigma_{ \pm}=\left(r^{2} \pm c+s\right) / 2>0 .
\end{aligned}
$$

In the first equation, $R_{C}\left(z^{2}, z^{2}+c\right)$ denotes the branch that is continuous in $c$ and takes the value $1 / z$ when $c=0$. In the exceptional cases where $z^{2}$ is real and $c / z^{2}<-1$, it denotes the Cauchy principal value of $(1 / z) R_{C}\left(1,1+c / z^{2}\right)$. On the right side of the first equation, each $R_{C}$ denotes the principal branch represented by (1.5).

\section{BIBLIOGRAPHY}

1. P. F. Byrd and M. D. Friedman, Handbook of elliptic integrals for engineers and scientists, 2nd ed., Springer-Verlag, New York, 1971.

2. B. C. Carlson, Special functions of applied mathematics, Academic Press, New York, 1977.

3. _ Elliptic integrals of the first kind, SIAM J. Math. Anal. 8 (1977), 231-242.

4. _ _ A table of elliptic integrals of the second kind, Math. Comp. 49 (1987), 595-606. (Supplement, ibid., S13-S17.)

5. __ A table of elliptic integrals of the third kind, Math. Comp. 51 (1988), 267-280. (Supplement, ibid., S1-S5.)

6. __ A table of elliptic integrals: cubic cases, Math. Comp. 53 (1989), 327-333.

7. Landen transformations of integrals, Asymptotic and Computational Analysis ( $R$. Wong, ed.), Marcel Dekker, New York, 1990, pp. 75-94.

8. _ A table of elliptic integrals: one quadratic factor, Math. Comp. 56 (1991), 267-280.

9. I. S. Gradshteyn and I. M. Ryzhik, Table of integrals, series, and products, Academic Press, New York, 1980.

ames Laboratory and Department of Mathematics, Iowa State University, Ames, IOWA 50011

E-mail address: bccarlson@decst2.ams.ameslab.gov 